\title{
ARTICLE
}

\section{Functional connectivity underpinnings of electroconvulsive therapy-induced memory impairments in patients with depression}

Danhong Wang ${ }^{1}$, Yanghua Tian ${ }^{2,3}$, Meiling $\mathrm{Li}^{1,4}$, Louisa Dahmani ${ }^{1}$, Qiang Wei ${ }^{2,3}$, Tongjian Bai ${ }^{2,3}$, Franziska Galiè ${ }^{1}$, Jianxun Ren ${ }^{1}$, Rai Khalid Farooq ${ }^{5}$, Kangcheng Wang ${ }^{1}$, Jie $\mathrm{Lu}^{6}$, Kai Wang ${ }^{2,3}$ and Hesheng Liu (iD ${ }^{1,7,8}$

Electroconvulsive therapy (ECT) is an effective treatment for severe medication-resistant depression. However, ECT frequently results in episodic memory impairments, causing many patients to discontinue treatment. The objective of this study was to explore the functional connectivity underpinnings of ECT-induced episodic memory impairments. We investigated verbal episodic memory and intrinsic functional connectivity in 24 patients with depression $(13 \mathrm{~F}, 11 \mathrm{M})$ before and after ECT, and 1 month after treatment. We used a novel individual-oriented approach to examine functional connectivity, and trained a linear support vector regression model to estimate verbal memory performance based on connectivity. The model identified a set of brain connections that can predict baseline verbal memory performance $(r=0.535, p=0.026)$. Importantly, we found a nonoverlapping set of brain connections whose changes after ECT can track patients' verbal memory impairments $(r=0.613, p=0.008)$. These connections mainly involve the frontoparietal control, default mode, and hippocampal networks, suggesting that ECT affects broad functional networks that are involved in memory performance. In contrast, functional connectivity defined using traditional group-level analyses was unable to estimate either baseline memory performance or post-ECT verbal memory impairments. A parallel analysis using the same strategy did not identify a connectivity marker for overall mood improvement, suggesting that functional connectivity changes related to depressive symptoms may be highly heterogenous. Our findings shed light on the mechanism through which ECT impairs episodic memory, and additionally underline the importance of accounting for interindividual variability in the investigation of functional brain organization in patients with depression.

Neuropsychopharmacology (2020) 45:1579-1587; https://doi.org/10.1038/s41386-020-0711-2

\section{INTRODUCTION}

Electroconvulsive therapy (ECT) as a treatment for depression can lead to remission rates as high as $80 \%$, making it one of the most effective treatments for this disease [1]. However, ECT often results in negative cognitive side effects, the most severe of which are episodic memory impairments [2-5]. While adverse cognitive effects usually subside weeks after ECT [6-8], some memory deficits can persist for months to years after treatment $[2,9]$.

The mechanism by which ECT induces adverse cognitive effects is not yet understood [3]. Most memory studies have investigated the state of the hippocampus as a potential mediating factor. For example, older adult patients with lower hippocampal volumes preceding ECT show worse memory-related side effects after treatment [10]. Several studies, including studies with large cohorts of patients from the Global ECT-MRI Research Collaboration, have reported increased hippocampal volume as a result of ECT treatment [11-16]. This volume change may be associated with the memory impairments observed after ECT [17].
An alternative mechanism by which ECT may exert negative effects on memory and more generally on cognition is through its modulation of large-scale functional brain networks rather than the hippocampal network alone. There is a growing body of evidence that many functional connectivity networks are dysregulated in depression [18-20], and ECT may alter connectivity in a way that contributes to patients' improved mood [21]. At the same time, connectivity changes across multiple functional networks may lead to memory impairments. Importantly, the exact connectivity underpinnings of memory impairments are yet to be revealed.

The mechanisms through which ECT exerts rapid antidepressant effects are also complex. Recent studies suggested that depression is a heterogeneous syndrome with different clinical features that do not map to the same brain circuit [22], thus there may not exist a single imaging marker for the ECT-induced mood improvement. Previous research has demonstrated that ECT can lead to anatomical and functional changes in a number of brain

\footnotetext{
${ }^{1}$ Athinoula A. Martinos Center for Biomedical Imaging, Department of Radiology, Massachusetts General Hospital, Harvard Medical School, Charlestown, MA, USA; ${ }^{2}$ Department of Neurology, The First Affiliated Hospital of Anhui Medical University, Hefei, China; ${ }^{3}$ Anhui Province Key Laboratory of Cognition and Neuropsychiatric Disorders, Hefei 230022 , China; ${ }^{4}$ Key Laboratory for Neurolnformation of Ministry of Education, School of Life Science and Technology, University of Electronic Science and Technology of China, Chengdu, China; ${ }^{5}$ Institute for Research and Medical Consultations, Imam Abdulahman Bin Faisal University, Dammam, Saudi Arabia; ${ }^{6}$ Department of Radiology, Xuanwu Hospital, Beijing,

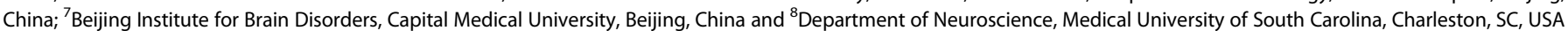
Correspondence: Kai Wang (wangkai1964@126.com) or Hesheng Liu (hesheng.liu@mgh.harvard.edu)

These authors contributed equally: Danhong Wang, Yanghua Tian, Meiling Li, Louisa Dahmani
}

Received: 10 December 2019 Revised: 20 April 2020 Accepted: 11 May 2020

Published online: 20 May 2020 
regions and these changes may be related to the antidepressant effects of ECT. For example, increased volume of the amygdala, hippocampus, and nucleus accumbens in response to ECT was found to be related to symptom improvement $[13,23]$. Graph theory analysis revealed that functional connectivity within a hippocampusthalamus-striatum network decreases in ECT responders [24]. Increased cerebral blood flow in regions such as the dorsomedial thalamus and motor cortex near the ECT electrode, and decreased cerebral blood flow in lateral frontoparietal regions have also been observed in ECT responders [25].

Here, using a subject-specific resting-state functional connectivity analysis, we examined these possible mechanisms and investigated how functional brain network changes induced by ECT affect episodic memory. Until recently, most imaging studies investigated the functional organization of the brain by using group-averaged data. However, it has been increasingly recognized that there is considerable interindividual variability in brain functional organization $[26,27]$, especially in regions involved in cognitive domains, including executive control and memory [26]. Thus, when investigating cognition, it is necessary to use methods that are able to capture individual differences in functional anatomy. Recent individual-oriented approaches in resting-state functional connectivity have been successful in this pursuit [28-31] and demonstrated great promise in clinical research [21,32].

Using a machine learning approach, we aimed to identify connections that could predict verbal memory performance in a sample of patients with depression prior to ECT treatment. We then sought to identify functional connections whose ECT-induced changes track memory impairments. We tested whether ECTinduced memory impairments are associated with connectivity changes within the networks responsible for memory processing at baseline, or whether they are associated with connectivity changes in broader functional networks. Finally, in a parallel analysis, we used a similar strategy to test whether there exists a functional connectivity signature for the ECT-induced mood improvement in our patient sample.

\section{MATERIALS AND METHODS}

\section{Participants}

Twenty-seven patients with DSM-IV diagnosed Major Depressive Disorder who showed resistance to medication were prescribed a course of ECT at the Anhui Mental Health Center. Our exclusion criteria were substance dependence, pregnancy, life-threatening somatic disease, neurological disorders, other co-morbid mental disorders, or MRI-related contraindications. Specifically, patients diagnosed with bipolar disorder were excluded. The diagnosis was conducted by two psychiatrists based on structured clinical interviews. In cases where there was disagreement between the two psychiatrists, patients were excluded from the study. All patients were taking antidepressants throughout the study, and medication was not adjusted during the four weeks preceding ECT. The categories of medicines taken by the patients are presented in Table 1. Treatment with benzodiazepines, valproate, and/or lithium was suspended during ECT administration. Patients underwent MRI scans and behavioral tests at three time points: 12-72 $\mathrm{h}$ before the first ECT, 1-7 days following the last ECT, and 1 month after the last ECT. At the first time point, three patients were excluded due to high level of head motion (mean relative head motion $>0.20 \mathrm{~mm}$ ) or low signal to noise ratio (slice-based temporal signal-to-noise ratio <100) [33], thus 24 patients (mean age $36.54 \pm 11.52$; 13 women, 11 men; all right handed) were included in the pre-ECT analyses. At the second time point, imaging data from three more patients were excluded for the same reasons. At the third time point, 14 patients were retested and three were excluded again after data quality control. The demographic information of the patients included at each time point is listed in Table 1 . The experiment was approved by the
Table 1. Patients' demographic and clinical information.

\begin{tabular}{llll}
\hline & $\begin{array}{l}\text { Visit 1 } \\
(n=24)\end{array}$ & $\begin{array}{l}\text { Visit 2 } \\
(n=21)\end{array}$ & $\begin{array}{l}\text { Visit 3 } \\
(n=11)\end{array}$ \\
\hline Age & $36.54 \pm 11.52$ & $36.38 \pm 11.79$ & $37.55 \pm 9.75$ \\
Gender & F: 13, M: 11 & F: 11, M: 10 & F: 5, M: 6 \\
Education & $8.92 \pm 3.62$ & $8.90 \pm 3.85$ & $8.91 \pm 4.32$ \\
Motion & $0.049 \pm 0.027$ & $0.066 \pm 0.031$ & $0.057 \pm 0.032$ \\
HAMD & $22.42 \pm 4.45$ & $3.81 \pm 2.25$ & $4.27 \pm 2.05$ \\
RAVLT & $6.71 \pm 3.14$ & $4.19 \pm 3.83$ & $9.36 \pm 3.56$ \\
Medications (number of subjects) & & \\
SNRIs & 10 & 9 & 6 \\
SSRIs & 14 & 14 & 5 \\
NaSSAs & 3 & 2 & 1 \\
TACs & 1 & 0 & 0 \\
SARls & 2 & 1 & 1 \\
Anticonvulsant & 3 & 3 & 0 \\
Non-benzodiazepine & 3 & 2 & 0 \\
hypnotic & & 1 & 1 \\
NRI & 1 & 10 & 3 \\
Antipsychotics & 8 & 4 & 3 \\
Antianxiety & 3 & & \\
\hline Values reprent means & & & \\
\hline
\end{tabular}

Values represent means \pm SD, or $n$.

Visit 1: 12-72 $\mathrm{h}$ before the first ECT; Visit 2: 1-7 days following the last ECT; Visit 3: 1 month after the last ECT. Data are shown as mean \pm standard deviation.

SNRIs serotonin-norepinephrine reuptake inhibitors, SSRIs selective serotonin reuptake inhibitors, NaSSAs norepinephrine and specificity serotonergic antidepressants, TCAs tricyclic antidepressants, SAR/s serotonin antagonist/ reuptake inhibitors, NRIs selective norepinephrine reuptake inhibitors.

Anhui Medical University Ethics Committee and was performed in accordance with all relevant guidelines and regulations. Written informed consent was obtained from all participants in accordance with the Anhui Medical University Ethics Committee.

\section{ECT procedures}

Modified bi-frontal ECT (maximum output: $1008 \mathrm{mC}$; Thymatron System IV Integrated ECT Instrument, Somatics Incorporation, Lake Bluff, IL, USA) was administered using a constant current of $0.9 \mathrm{~A}$ with $1.0 \mathrm{msec}$ pulse width, after induction of anesthesia intravenously with propofol $(0.2-0.5 \mathrm{mg} / \mathrm{kg}$ body mass), muscle relaxation with succinylcholine $(0.5-1 \mathrm{mg} / \mathrm{kg}$ body mass), and suppression of gland secretion intravenously with atropine $(0.5-1 \mathrm{mg} / \mathrm{kg}$ body mass). Seizure activity was monitored with electroencephalography. A minimum of six ECT sessions were administered. The first three ECT administrations occurred on consecutive days, and the remaining ECT administrations were conducted every other day with a break on weekends until patients' symptoms remitted. Previous studies suggested that lower frequency treatment would necessitate a longer treatment to alleviate mood symptoms, however this concomitantly results in more severe cognitive impairments [34]. Age is also an important factor when determining the treatment frequency. Elderly people are more prone to cognitive impairments following ECT, and should therefore undergo lower frequency treatment. Our sample was mostly comprised of young patients (mean age $36.54 \pm 11.52$ ). Thus, in this study we administered three daily sessions at the beginning, according to the guidelines of the Chinese Association of Physicians for Electrical Shock and Nerve Stimulation, which recommends transient use of daily treatment for younger patients (Electroconvulsive Therapy Expert Consensus: 2017 Edition, Chinese Association of Physicians for Electrical Shock and Nerve Stimulation). Remission was defined 
as a 17-item Hamilton Depression Rating Scale (HAMD) [35] score of 7 points or less. Two independent experienced psychiatrists administered the HAMD. If patients did not remit after six sessions, treatment continued until remission was achieved or until a maximum of $12 \mathrm{ECT}$ sessions have been administered. On average, patients received $6.87 \pm 2.24 \mathrm{ECT}$ sessions.

Initial percent energy dial setting threshold was measured at the first ECT session based on the age of the patient. If the patient was older than 50 years old, the initial percent energy dial was set at the patient's age (For example, 53\% for a 53-year-old patient). If the patient was under 50 years old, the initial percent energy dial was set at the patient's age minus five (for example, $40 \%$ for a 45 year-old patient). If no seizure activity resulted, the percent energy was increased until a therapeutically satisfactory seizure was obtained. The mean stimulus intensity was $37.11 \%$ (from 15 to $80 \%)$ and the mean pulse frequency was $34.03 \mathrm{~Hz}(\mathrm{SD}=10.16)$.

Clinical and neuropsychological measures

A battery of clinical and neuropsychological tests was administered, including the 17-item HAMD [35], Mini Mental State Exam [11-16], and Rey-Auditory Verbal Learning Test (RAVLT) [36]. Details about these tests can be found in Supplementary Materials and Methods.

MRI data acquisition and preprocessing

All MRI data were acquired on a 3.0 T whole-body GE MRI scanner (Signa HDxt 3.0 T, GE Healthcare, Buckinghamshire, UK) at the First Affiliated Hospital of Anhui Medical University. T1-weighted anatomical images were acquired in sagittal orientation with three-dimensional inversion recovery prepared fast spoiled gradient recalled sequence (repetition time/echo time ratio $=$ $8.676 / 3.184 \mathrm{~ms}$, inversion time $=800 \mathrm{~ms}$, flip angle $=8$ degrees, field of view $=256 \times 256 \mathrm{~mm}^{2}$, matrix size $=256 \times 256$, slice thickness $=1 \mathrm{~mm}$, voxel size $=1 \times 1 \times 1 \mathrm{~mm}^{3}, 188$ slices). Functional MRI (fMRI) BOLD images were acquired with the following parameters: repetition time/echo time ratio $=2000 / 22.5 \mathrm{~ms}$, flip angle $=30$ degrees, 33 slices, thickness $/$ gap ratio $=4.0 / 0.6 \mathrm{~mm}$, voxel size $=3.4 \times 3.4 \times 4.6 \mathrm{~mm}^{3}$, matrix size $=64 \times 64$, field of view $=220 \times 220 \mathrm{~mm}^{2}$. Participants were instructed to stay awake, keep their eyes open, and minimize head movement.

Structural MRI data were preprocessed using FreeSurfer version 5.3.0 as described by Yeo et al. [37]. Resting-state fMRI data were preprocessed using previously described procedures [37] that were adapted from Van Dijk et al. [38]. The following steps were performed for $\mathrm{fMRI}$ preprocessing: (1) slice timing correction using SPM (SPM2; http://www.fil.ion.ucl.ac.uk/spm/); (2) rigid body correction for head motion using FSL (https://fsl. fmrib.ox.ac.uk/fsl/fslwiki/); (3) band-pass temporal filtering (0.01 $\mathrm{Hz}-0.08 \mathrm{~Hz}$; (4) spurious variance and their derivatives were moved through regression that included head motion, the average signal within a ventricle mask, and the average signal within a white matter mask. Whole-brain signal regression was also included in the preprocessing stream to improve the correction of motion-related artifacts $[39,40]$. In the present study, we did not censor the image frames based on head motion because our previous exploration indicated that the data scrubbing might cause inflated connectivity estimates in specific regions [41]. Surface mesh representations of the cortex from each individual participant's structural images were reconstructed and registered to a common spherical coordinate system. The structural and functional images were aligned using boundary-based registration within the FsFast software package (http://surfer.nmr.mgh.harvard.edu/fswiki/FsFast). The preprocessed resting-state BOLD $\mathrm{FMRI}$ data were normalized to the MNI space to yield volumetric time series, and also aligned to the common spherical coordinate system to derive surface time series for cerebral cortex. A 6-mm full-width half-maximum smoothing kernel was applied to the fMRI data in the surface space and then the data were downsampled to the fsaverage4 mesh with 2562 vertices in each hemisphere.

Population-level functional atlas

A population-level functional atlas including 17 cortical networks was obtained using data from 1000 healthy participants [37]. We further separated the hand areas, a common area mapped in surgical patients, from this atlas based on the activations from a hand motor task [28, 42]. As a result, this population atlas consisted of 18 networks (Fig. S1), and was divided into 116 discontinuous regions of interest (ROls) (see Supplementary Materials and Methods for more details). The 116 ROls from within the 18 networks can be further assigned into 7 canonical networks: visual network (VIS), sensorimotor network (MOT), limbic network (LMB), frontoparietal control network (FPN), dorsal attention network (ATN), salience network (SAL), and default mode network (DMN) [37].

Identifying homologous functional ROIs in individuals The population-level cortical ROls identified above were used as an initial functional template, following which homologous ROls were identified in each individual, according to the steps described in our previous reports $[28,31]$. First, we mapped 18 cortical networks in each participant by applying an iterative, individualized parcellation approach [28]. We then segmented the individually defined cortical networks into discrete "patches" using a clustering algorithm (mri_surfcluster in FreeSurfer). The discrete patches within each participant were labeled using the approach described in [31] (see Supplementary Materials and Methods for more details).

ECT-induced changes in the functional connectivity of functional networks

To assess the changes in functional connectivity induced by ECT, we calculated the dissimilarity between the pre- and post-ECT functional connectivity profiles of each individualized cortical ROI and subcortical ROI. The connectivity profile of a given ROI was defined as the connectivity between the seed ROI and the remaining ROls. For each $\mathrm{ROI}$, we first calculated the correlation between pre- and post-ECT functional connectivity and then the correlation coefficient $r$ was subtracted from 1. Dissimilarity was estimated as $(1-r)$. To determine the statistical significance of the dissimilarity and to correct for multiple comparisons, we used a false discovery rate (FDR)-corrected threshold of $q=0.005$.

\section{Symptom estimation}

Among the 116 cortical ROls, we were able to identify 84 homologous functional ROls across all participants using the procedure described above. To date, a reliable technique for mapping functional networks in individual participants' subcortical regions is not yet available. However, given the critical role of subcortical regions in depression $[43,44]$, we added 22 predefined subcortical ROls in the present study, which were centered in: seven left/right subregions of thalamus, left/right amygdala, left/right caudate nucleus, left/right hippocampus, and left/right putamen. Two separate functional connectivity matrices based on 106 individual- and population-level ROls (i.e., 84 regions on the cortex and 22 subcortical regions) were generated for each individual for the exploration of connectivitybehavior relationships.

We trained a support vector machine for regression (SVR) model to estimate each patient's RAVLT delayed recall and HAMD score based on functional connectivity. We used pre-ECT functional connectivity to estimate baseline symptom scores, and post-pre ECT functional connectivity to estimate changes in symptoms. We did not investigate post-ECT functional connectivity alone in relation to memory impairment (RAVLT score) or mood improvement (HAMD score) as ECT-induced network disruption was likely 
to differ widely from patient to patient, making it unlikely for the SVR model to detect a specific pattern of disruption. Post- vs. preECT functional connectivity is a more robust analysis for this investigation as it is longitudinal and captures changes in functional connectivity within individual patients.

We also sought to determine whether functional connectivity changes in the month following ECT could track memory changes. To do so, we focused on the connections that were associated with memory impairments in the post-pre ECT analyses, and measured the changes in these connections from the post-ECT scan to the 1-month follow-up scan.

The L2-regularized L2-loss SVR model implemented in the LIBLINEAR package (https://www.csie.ntu.edu.tw/ cjlin/liblinear/) was applied. We employed the leave-one-out cross validation method, e.g. data from $\mathrm{N}-1$ participants were used to train the model and the output model was applied to the remaining participant's data to estimate the participant's symptom scores. We repeated this procedure $N$ times to estimate all participants' symptom scores. Correlations between the estimated and observed symptom scores were then calculated. We performed nonparametric permutation tests (1000 permutations) to verify whether the correlation was a product of chance. The permutation $p$ value was determined as the percentage of permutations that resulted in a correlation $r$ coefficient higher than the correlation based on the real data. To decrease the dimensionality of the input, in each LOOCV fold, we selected a subset of connections based on their correlation to symptom scores in the training sample [45].

Calculating the contribution of each network and ROI to symptom score estimation

In the symptom score estimation models, some cortical connections may have contributed more greatly to the estimation than other connections. In the model, a connection's contribution was quantified by its "weight". For each LOOCV fold, the SVR model calculated a weight coefficient for each feature in the training data. For any given connection, its contribution to the symptom score estimation was then calculated by averaging its weight across all LOOCV folds. A connection's contribution was set to zero if the connection was not selected as the feature in a given fold. ROI contributions were calculated by summing up the contributions of all the connections that were involved therein.

In order to quantify the contribution of each functional network to the symptom score estimation, homologous functional ROls were grouped into the seven canonical functional networks [37]. We separated between- from within-network connections according to whether the connections stemmed from two ROls within the same network or different networks. For each network, within-network and between-network weights were estimated. For the within-network contributions, the connection weights within a given network were added up. For the between-network contributions, weights of all connections that involved an ROI within the network and an ROI outside of the network were added up.

\section{Visualization}

For visualization, the imaging results were mapped onto the inflated PALS cortical surface with CARET [46]. The connectograms in Figs. 1 and 2 showing connections contributed to RAVLT score estimation were made with Circos (http://circos.ca/).

\section{RESULTS}

ECT led to improvement in mood but caused significant verbal memory impairments

Comparing the depression symptoms in the 24 patients who were examined before and after ECT treatment, we found a significant decrease in HAMD score $(22.22 \pm 4.74$ vs. $3.83 \pm 2.15, p<0.0001$, $t=17.19$, paired $t$-test). RAVLT delayed recall scores showed a concurrent and significant reduction following ECT treatment $(6.83 \pm 3.16$ vs. $3.91 \pm 3.78, p=0.001, t=3.66$, paired $t$-test), indicating that ECT is effective in reducing depression symptoms but simultaneously leads to significant memory impairments. Interestingly, RAVLT score decrease was uncorrelated with HAMD score reduction $(r=-0.07, p=0.742)$, suggesting that memory impairment and mood improvement are due to separate biological mechanisms. This is corroborated by the observation that, in a subset of patients $(n=17)$ who were examined 1 month after ECT treatment, HAMD scores remained at a low level compared to baseline $(23.06 \pm 3.85$ at baseline vs. $4.12 \pm 2.06$ at follow-up, $p<0.0001, t=18.44$, paired $t$-test), whereas memory performance improved significantly after 1 month $(6.71 \pm 3.22$ at baseline vs. $8.94 \pm 3.11$ at follow-up, $p=0.004, t=-3.35$, paired $t$-test).

ECT affects the functional connectivity of widespread functional networks

ECT induces non-focal seizures, and therefore we expected it to affect networks widely distributed over the brain. To reveal what brain regions exhibit more prominent changes than other regions, we quantified the dissimilarity between pre- and post-ECT functional connectivity profiles for each of the 84 cortical and 22 subcortical regions (see Methods). Widespread brain regions showed significant changes following ECT (Fig. 3, FDR corrected, $q=0.005$ ), including some brain areas that are relevant to memory and mood. Some regions of the DMN and limbic networks exhibited particularly strong changes in their functional connectivity profiles, including the medial temporal lobe, the hippocampus, precuneus, and subgenual cingulate area.

Individual-specific functional connectomes track baseline verbal memory

We sought to determine whether individual differences in verbal memory performance can be predicted by subject-specific functional connectivity. Functional connectivity was calculated among subcortical and individualized cortical ROls for each individual. We trained SVR models to estimate the pre-ECT RAVLT scores based on connectivity from each of the 24 patients who had baseline fMRI scans. A significant correlation was found between the estimated and observed RAVLT scores $(r=0.535, p=$ 0.026; Fig. 1a), indicating that a set of functional connections can robustly estimate verbal memory performance. The connections that contributed most to the estimation of RAVLT scores involved the FPN, DMN, and VIS, as well as the subcortical regions (Fig. 1b). We then repeated the estimation analysis using functional connectivity among 116 ROls defined by a population-level atlas [37] and found that the functional connectivity model was unable to estimate RAVLT scores ( $r=-0.496, p=0.874$, Fig. $1 c)$, indicating that this brain-behavior association was missed when individual differences in functional anatomy were not properly accounted for.

Changes in individual-specific functional connectomes track verbal memory impairment following ECT

We next tested whether the functional connectivity could track memory impairments in 21 patients who had sufficient pretreatment and posttreatment imaging data. To do this, we trained SVR models to estimate the changes in RAVLT scores based on the changes in functional connectivity. We again found that an individual-based model robustly estimates the changes in RAVLT scores, with a statistically significant correlation between estimated and observed changes ( $r=0.613, p=0.008$; Fig. 2a). The networks that most contributed to the estimation model include the FPN, DMN, MOT, SAL, as well as the left hippocampus (Fig. 2b). In contrast, the group-level approach did not yield a model that could estimate the changes in RAVLT scores, as indicated by a 

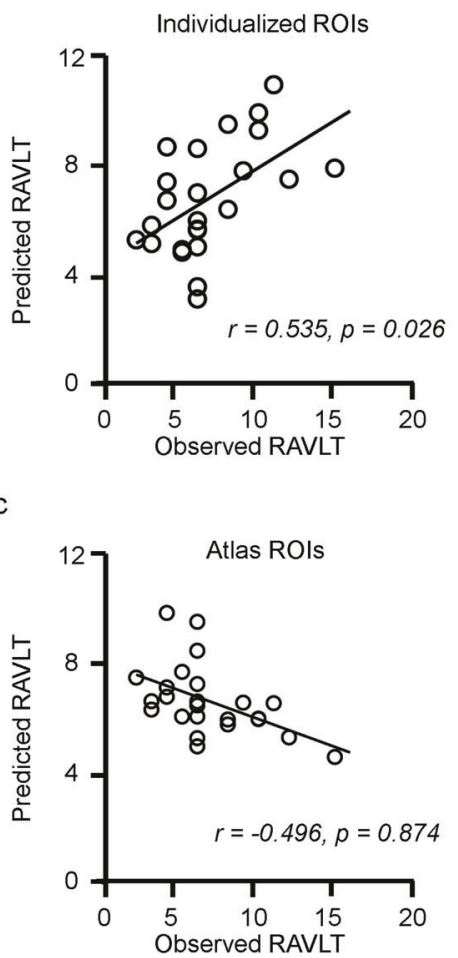

b

Connections predicting baseline RAVLT
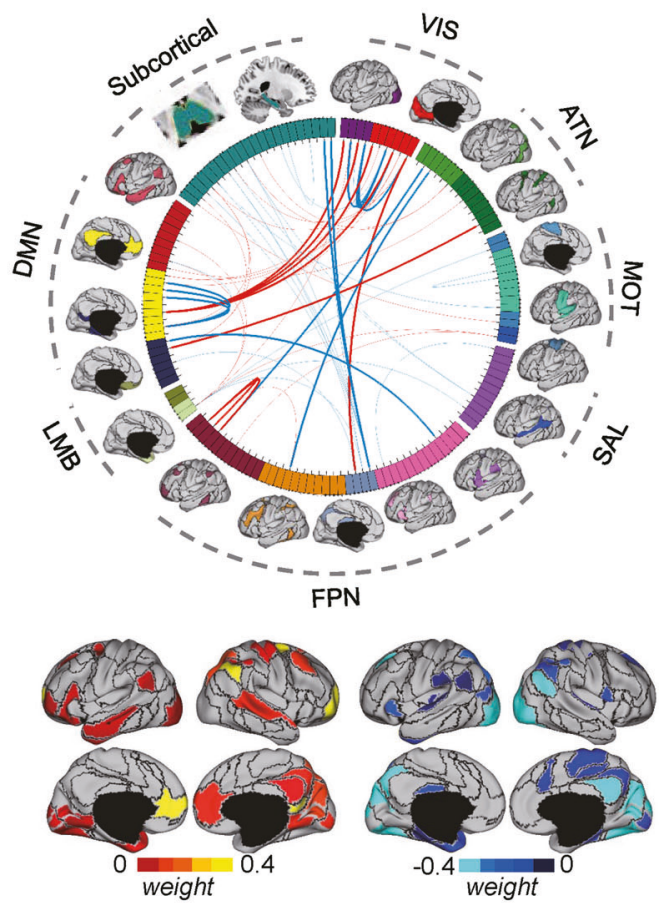

Fig. 1 Functional connectivity among individually specified ROIs can estimate baseline verbal memory scores before ECT treatment. a Correlation ( $r=0.535, p=0.026$, permutation test) between the RAVLT scores estimated by connectivity among the individually specified ROIs and the scores that were actually observed in the 24 patients before ECT treatment. $\mathbf{b}$ Connections contributing to estimate the pretreatment RAVLT. One hundred and sixteen cortical regions extracted from 18 networks and 22 subcortical regions are represented on a wheel. Group-level maps of the 18 functional networks and subcortical regions (e.g., thalamus, hippocampus) are shown outside the wheel. These ROls are colorcoded according to the seven canonical networks: visual (VIS), sensorimotor (MOT), attention (ATN), salience (SAL), limbic (LMB), frontoparietal control (FPN), default mode network (DMN), and the subcortical network. Fifty-eight connections that are predictive of RAVLT scores are plotted (top 20 connections are indicated by thick lines). Connections that were positively correlated with RAVLT scores are shown in red and connections that were negatively correlated with RAVLT scores are shown in blue. Functional regions involved in these two groups of connections are rendered on the cortical surface. c A similar analysis is performed using ROls defined in a group-level network template. Functional connectivity based on the brain atlas is not able to predict RAVLT scores in patients $(r=-0.496, p=0.874$, permutation test).

nonsignificant and negligible correlation between estimated and observed changes in RAVLT scores, $(r=-0.067, p=0.740$, Fig. 2c). Critically, connections that contributed to the baseline memory estimation models showed almost no overlap (Dice's coefficient $=0.09$ ) with the connections that contributed to memory impairment estimation models (Fig. 4a), indicating that ECT-induced memory impairments are not due to changes in the brain connections that were associated with baseline memory. However, many of the same regions are involved in both models, including the hippocampus and regions in the DMN, ATN, and FPN networks, which have all previously been shown to be involved in memory [47-49].

ECT-induced memory impairment is mainly due to changes in between-network connectivity

We then looked at within- and between-network connections (see Methods) that were identified by the SVR model in estimating the baseline verbal memory and ECT-induced verbal memory impairments. The between-network connections associated with baseline memory performance involved the FPN, subcortical, and DMN, while within-network connections mostly involved the FPN, DMN, and VIS (Fig. 4b). When looking at within- vs. between-network connections associated with ECT-induced memory impairments, we found that these connections were mostly comprised of between-network connections. The between-network connections involved the FPN, DMN, MOT, and the subcortical regions (hippocampus and thalamus). The within-network connections were negligible (Fig. 4c, also see Fig. 2b).

We investigated how the individual specification of functional regions impacted the SVR model. First, the individual-specific connections (e.g., Figs. $1 \mathrm{~b}$ and $2 \mathrm{~b}$ ) contributing to the prediction of baseline memory and ECT-induced memory impairments were redefined using group-level ROIs. We found that these atlas-based connections were less correlated with memory scores compared with individual-specific connections (Fig. S2, $p<0.001, t=5.94$ for baseline memory and $p<0.001, t=9.01$ for memory impairment, paired $t$-tests). This indicates that the connectivity-behavior associations were already obscured by the group-level atlas before the SVR model was applied, leading to a reduced prediction accuracy of the model. Second, we found that the absolute values of between-network connections were significantly reduced (average decrease of $12.51 \%, p<0.001$ for 17 out of the 18 networks), when ROls were individually specified than atlas-defined (Fig. S3). Intriguingly, although the absolute values of between-network connectivity were significantly reduced, they can yield better symptom estimates, suggesting that connections may be more accurately quantified when functional regions are localized in individuals.

Functional connectivity changes in the month following ECT track verbal memory improvement

Focusing on the connections that were associated with ECTinduced memory impairments (defined in the Fig. 2 analysis), we 

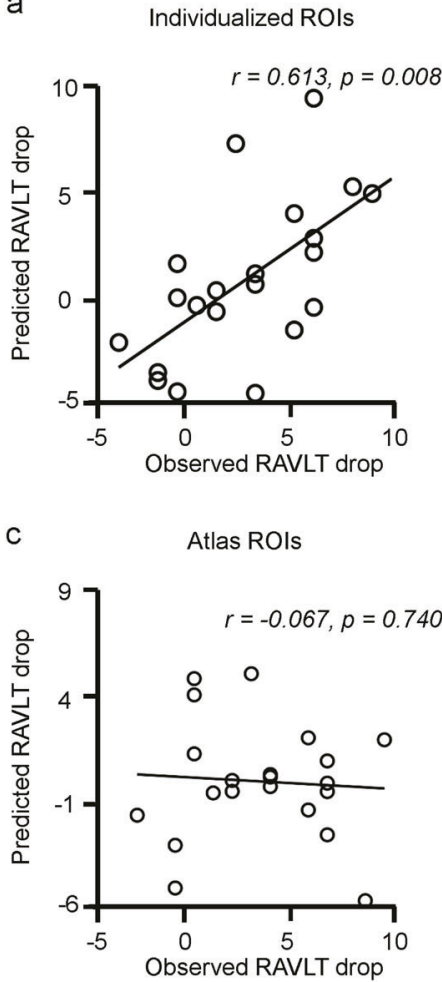

b

$$
\text { Connections predicting ECT-induced }
$$
impairments in RAVLT

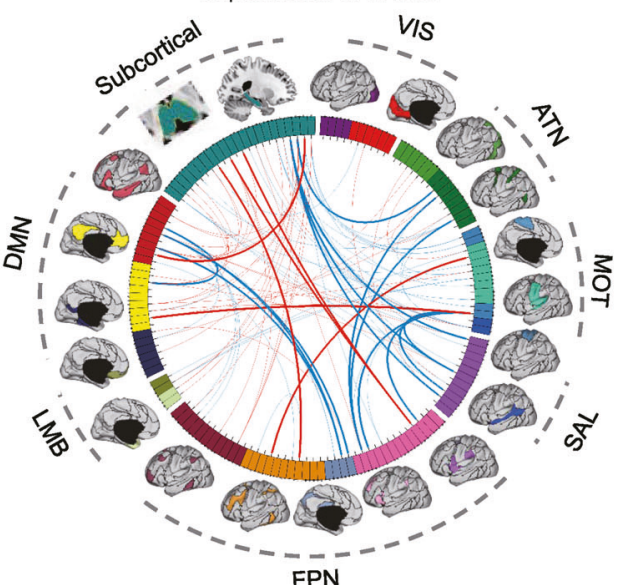

FPN
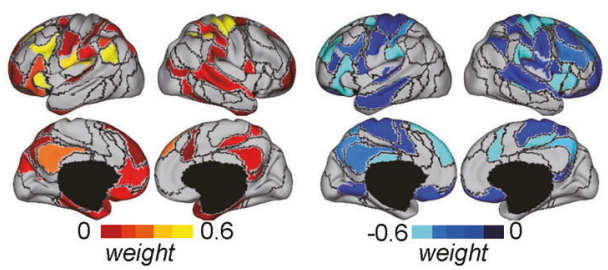

Fig. 2 Functional connectivity changes among individually specified ROIs track verbal memory impairments due to ECT. a Changes in the individually specified functional connectivity induced by ECT can estimate the changes in verbal memory scores (RAVLT change) following ECT $(r=0.613, p=0.008)$. b One hundred and one connections that are predictive of the verbal memory impairment are plotted on the wheel. Connectivity changes that were positively correlated with RAVLT score changes are shown in red and connectivity changes that were negatively correlated with RAVLT score changes are shown in blue (top 20 connections are indicated by thick lines). c Again, functional connectivity based on the brain atlas is not able to track verbal memory impairment $(r=-0.067, p=0.740$, permutation test).

Regions showing significant connectivity change post-ECT
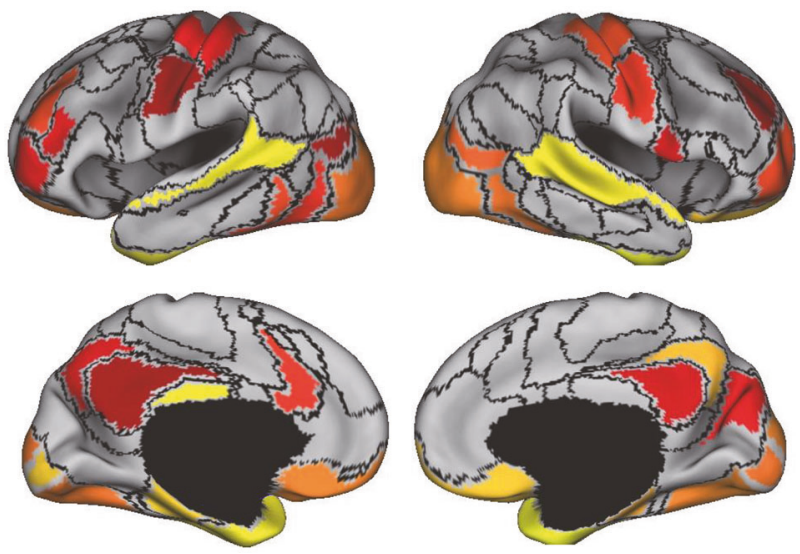

$$
0.15 \text { Dissimilarity }(1-r)
$$

Fig. 3 ECT affects the functional connectivity of widespread functional networks. The dissimilarity between pre- and post-ECT functional connectivity profiles was calculated for each of the 84 cortical and 22 subcortical ROIs. The colors represent regions whose functional connectivity profiles significantly changed (i.e., less correlated thus a higher $1-r$ ) from pre- to post-ECT (FDR corrected, $q=0.005$ ). Widespread cortical regions were altered by ECT, as well as the hippocampus (not pictured). Regions of the DMN and limbic network were especially affected. sought to determine whether the change in these connections in the month following the ECT treatment could track the improvement of RAVLT scores over that period. We found a trend in the correlation between estimated and observed improvement in RAVLT scores $(r=0.504, p=0.076$; Fig. S4), suggesting that a subset of connections may have recovered from the impairment after 1 month.

Individual-specific functional connectomes failed to estimate HAMD scores

We used a similar approach to examine the relationship between functional connectivity and depression symptoms, determining whether the SVR model can predict baseline HAMD scores or changes in HAMD scores following ECT using functional connectivity. The SVR model, whether based on individually specified or atlas-based functional connectivity, failed to predict either of these (Fig. 5; all $p^{\prime} \mathrm{s}>0.1$ ).

Additional analyses

We ensured that head motion did not significantly impact our results. RAVLT scores were uncorrelated with head motion $(p>0.1)$. We were also not able to directly predict head motion using functional connectivity $(p>0.1)$, indicating that our prediction model was unlikely to have been influenced by head motion (Fig. S5).

\section{DISCUSSION}

ECT shows great efficacy in treating depression symptoms but produces a significant decline in episodic memory. In this study, 


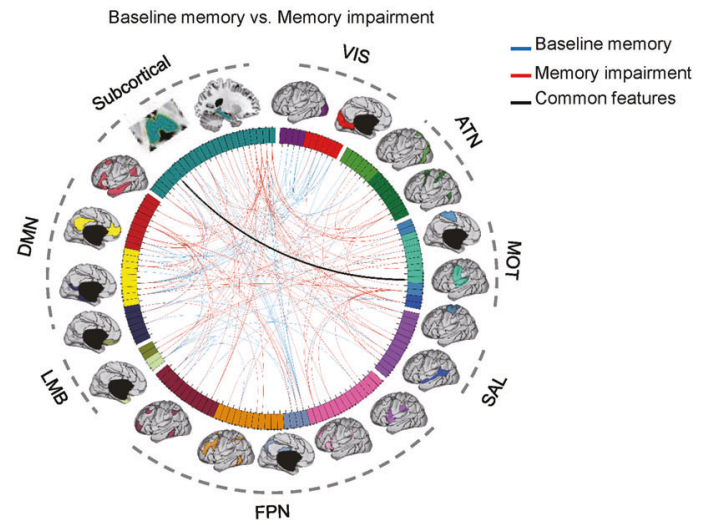

b
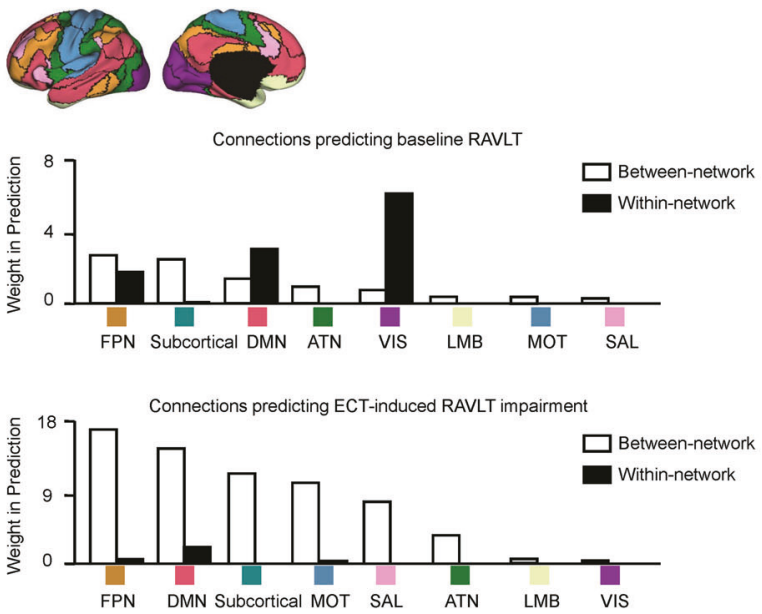

Fig. 4 Connections involved in baseline memory and memory impairment. a Individual-specific functional connections that contributed to estimating verbal memory scores in pretreatment (blue lines) and verbal memory impairment following ECT treatment (red lines) were largely nonoverlapping. b The functional connections most predictive of verbal memory scores are grouped into one subcortical and seven canonical networks. Connections contributing to the baseline verbal memory scores estimation are composed of within- and between-network connections. The between-network connections mainly involve the FPN, subcortical and DMN, while within-network connections mainly involve the VIS, DMN, and FPN. c Connections contributing to verbal memory impairment prediction mostly involve between-network connectivity in the FPN, DMN, and subcortical structures.

we investigated the functional connectivity underpinnings of ECT-induced verbal memory impairments. To properly account for the interindividual variability in functional anatomy, we employed an individual-specific approach to localize functional regions and then constructed individualized connectomes for brain-behavior analyses. We found that ECT affects widespread functional networks in the brain, altering the functional connectivity of brain regions that are relevant to memory and mood. Using machine learning, we identified a set of functional connections that track ECT-induced verbal memory impairments. These connections mainly involve the FPN, DMN, and hippocampus, and show minimal overlap with the connections associated with baseline memory performance, although many brain regions are commonly involved. Further analyses demonstrated that connections that track memory impairments are mostly between-network connections. However, a parallel analysis using the same strategy failed to identify a connectivity marker for overall mood improvement. Taken together, our data suggest that ECT-induced verbal memory impairments are due to altered communication among broad networks including the
FPN, DMN, and subcortical structures, rather than disruptions restricted to specific regions.

Memory impairment and mood improvement after ECT are due to separate biological mechanisms

Immediately following the conclusion of the ECT treatment, patients exhibited a marked improvement in mood. Concurrent with this, however, was a decrease in verbal memory. There was no correlation between mood improvement and verbal memory decline, suggesting that these changes are due to distinct biological mechanisms. In addition, while improved mood remained unchanged 1 month after ECT treatment, verbal memory improved, further supporting a separate neurobiological basis for limbic and cognitive effects of ECT.

Although we were able to identify connections that track verbal memory performance, we failed to identify a connectivity marker for mood improvement. Depression is known to be a highly heterogenous syndrome and functional connectivity patterns can vary substantially across different subtypes of depression [50]. We thus speculate that there may not exist a single connectivity marker for global depressive symptomatology but that instead each symptom dimension may have its unique biological substrate. This was supported by a recent Transcranial Magnetic Stimulation (TMS) study that identified two clusters of depressive symptoms that respond to two distinct neuroanatomical treatment targets [22]. Importantly, optimal TMS treatment targets for these two symptom clusters appear to be located in two competing functional networks. Future imaging studies may thus seek to identify the functional connectivity underpinnings of each symptom domain.

ECT affects broad networks that are related to memory performance

Using individualized connectomes, we found a set of functional connections that can track ensuing verbal memory impairments. These connections involve broad networks, mostly the FPN, DMN, and subcortical structures including the hippocampus (Fig. 1). Moreover, verbal memory impairments were mainly associated with between-network rather than within-network changes in functional connectivity (Fig. 4). A particular intriguing observation is the modulation of the FPN by ECT. A large-scale brain system implicated in complex cognition and contextual processing, the FPN is situated at the interface between the spatial attention network and the DMN, a system thought to be responsible for selfreferential information processing and memory. Recent studies provided ample evidence that the FPN may serve as a control system that mediates information processing between the external and internal worlds [51]. Bi-frontal ECT may thus directly impact the FPN and modulate its connectivity with the DMN, leading to changes in memory performance. In addition, our model revealed that ECT modulates the functional connectivity between the hippocampus and salience network. Based on the hippocampus's role in processing contextual information in episodic memory $[52,53]$, we speculate that this connectivity may be important in evaluating the saliency of events within the contexts they are ascribed in, and that altered connectivity would lead to episodic memory impairments. Collectively, these findings suggest that ECT affects interactions between a variety of functional networks that directly or indirectly impact memory performance.

Interindividual differences in functional anatomy is a critical factor in the investigation of ECT effects on cognitive functions We showed that functional connectivity defined using the individually specified functional regions was able to predict baseline verbal memory performance and ECT-induced verbal memory impairments to a certain extent. Critically, the same models using connectivity derived from a group-level atlas 


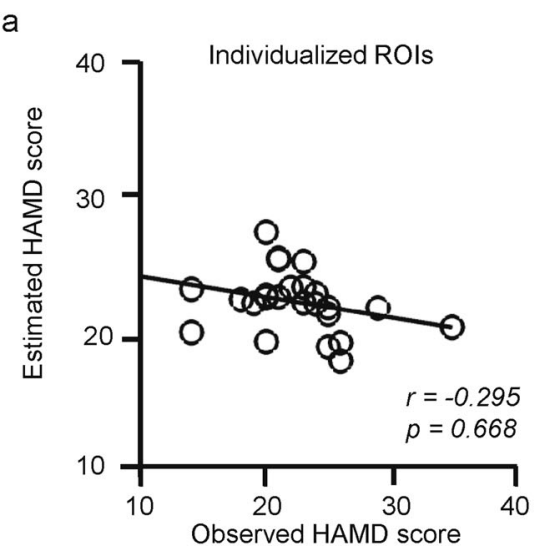

b

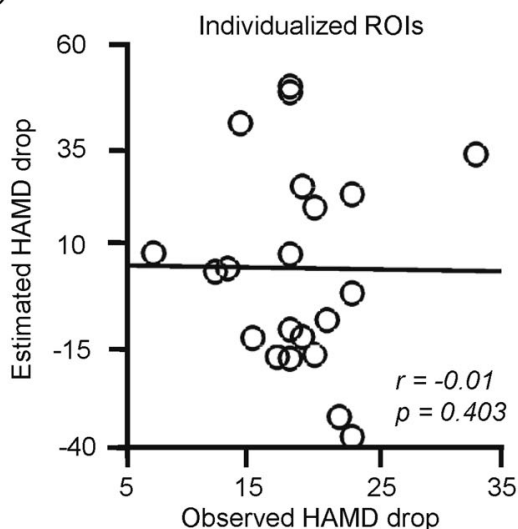

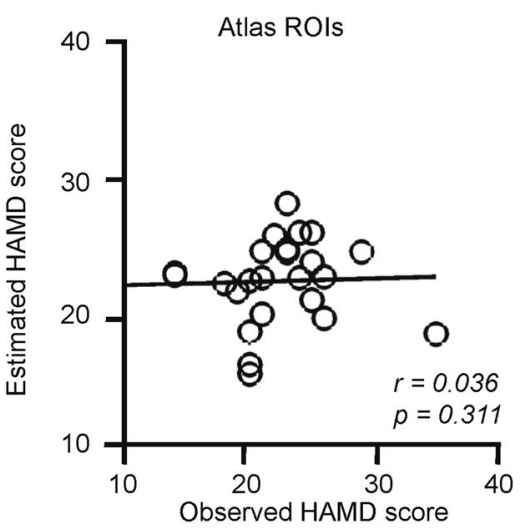

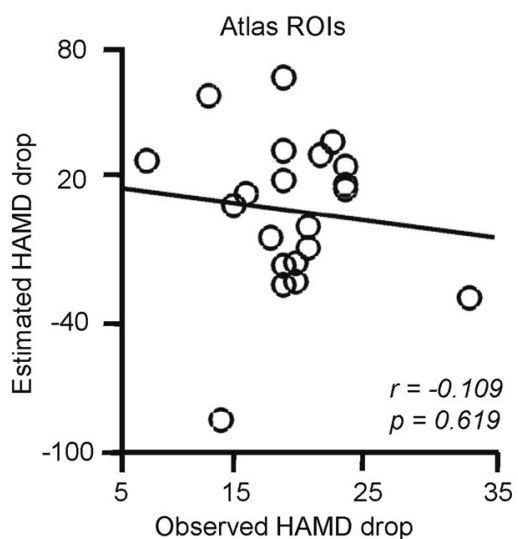

Fig. 5 Estimating depressive symptoms based on connectivity. Models based on individual and atlas-based functional connectivity failed to estimate either (a) baseline HAMD scores (individual-based: $r=-0.295, p=0.668$; atlas-based: $r=0.036, p=0.311$ ), or (b) mood improvement following ECT (individual-based: $r=-0.01, p=0.403$; atlas-based: $r=-0.109, p=0.619$ ).

completely lost the ability to predict these behavioral measures (Figs. 1c and 2c). Further analyses indicated that associations between connectivity and behavior measures were already significantly reduced in the group-level analysis even before any machine learning approaches were applied (Fig. S2). Frontal ECT is likely to modulate networks that are known to be most variable across individuals, including the FPN [26]. Thus, accounting for interindividual variability in functional anatomy appears to be a necessary step in future functional studies of ECT effects.

Limitations and future directions

The fact that ECT affected most of the broad functional brain networks makes it difficult to interpret the concrete ways in which verbal memory was impaired, although we propose that some connections among FPN, DMN, and the hippocampus may have been affected most. Future work should include an extensive battery of neuropsychological tests to better describe the functional connectivity effects of ECT on memory and cognition. In addition, there is a need for longitudinal studies of ECT effects. As it is unclear how long episodic memory impairments remain after ECT treatment [2, 6-9], it would be informative to determine how the functional organization of the brain evolves in the months following ECT and how these changes interact with mood and memory.

\section{CONCLUSION}

Our findings indicate that ECT induces changes in connectivity between a variety of functional networks that together account for memory impairments. Thus, ECT does not only modulate single regions, but large-scale networks and their connectivity, with acute effects on cognition. This work highlights the potential for a new functional connectomic biomarker of memory impairment following ECT.

\section{FUNDING AND DISCLOSURE}

This work was supported by the National Natural Science Foundation of China grant No. 81790650, 81790652, 81671354; $\mathrm{NIH}$ grants R01NS091604, P50MH106435 and K01MH111802. HL is on scientific advisory boards for NeuralGalaxy LLC, which is unrelated to the present work. The authors declare no conflict of interest.

\section{AUTHOR CONTRIBUTIONS}

$\mathrm{HL}$ and $\mathrm{KW}$ conceived the study; DW, YT, ML, QW, TB performed the study; ML, LD, $D W, Q W, T B, J R, F G$, and KW performed the analyses with support from JL and $H L$; LD, $H L$, DW, RKF, and FG wrote the paper with contribution from $J L$ and KW. All authors commented on the paper.

\section{ADDITIONAL INFORMATION}

Supplementary Information accompanies this paper at (https://doi.org/10.1038/ s41386-020-0711-2).

Publisher's note Springer Nature remains neutral with regard to jurisdictional claims in published maps and institutional affiliations. 


\section{REFERENCES}

1. Lisanby SH. Electroconvulsive therapy for depression. N Engl J Med. 2007; 357:1939-45.

2. Donahue $A B$. Electroconvulsive therapy and memory loss: a personal journey. J ECT. 2000;16:133-43.

3. Fraser LM, O'Carroll RE, Ebmeier KP. The effect of electroconvulsive therapy on autobiographical memory: a systematic review. J ECT. 2008;24:10-7.

4. Squire $L$, Slater $P$, Chace $P$. Retrograde amnesia: temporal gradient in very long term memory following electroconvulsive therapy. Science. 1975;187:77-9.

5. Squire LR. Memory functions six to nine months after electroconvulsive therapy. Arch Gen Psychiatry. 1975;32:1557.

6. Semkovska M, McLoughlin DM. Objective cognitive performance associated with electroconvulsive therapy for depression: a systematic review and meta-analysis. Biol Psychiatry. 2010;68:568-77.

7. Bodnar A, Krzywotulski M, Lewandowska A, Chlopocka-Wozniak M, BartkowskaSniatkowska $A$, Michalak $M$, et al. Electroconvulsive therapy and cognitive functions in treatment-resistant depression. World J Biol Psychiatry. 2016;17:159-64.

8. Squire LR, Miller PL. Diminution of anterograde amnesia following electroconvulsive therapy. Br J Psychiatry. 1974;125:490-95.

9. Sackeim HA, Prudic J, Fuller R, Keilp J, Lavori PW, Olfson M. The cognitive effects of electroconvulsive therapy in community settings. Neuropsychopharmacology. 2007;32:244-54

10. Lekwauwa R, McQuoid D, Steffens DC. Hippocampal volume is associated with physician-reported acute cognitive deficits after electroconvulsive therapy. J Geriatr Psychiatry Neurol. 2006;19:21-25.

11. Nordanskog P, Dahlstrand U, Larsson MR, Larsson E-M, Knutsson L, Johanson A. Increase in hippocampal volume after electroconvulsive therapy in patients with depression: a volumetric magnetic resonance imaging study. J ECT. 2010;26:62-67.

12. Tendolkar I, van Beek M, van Oostrom I, Mulder M, Janzing J, Voshaar RO, et al. Electroconvulsive therapy increases hippocampal and amygdala volume in therapy refractory depression: a longitudinal pilot study. Psychiatry Res: Neuroimaging. 2013;214:197-203.

13. Joshi SH, Espinoza RT, Pirnia T, Shi J, Wang Y, Ayers B, et al. Structural plasticity of the hippocampus and amygdala induced by electroconvulsive therapy in major depression. Biol Psychiatry. 2016;79:282-92.

14. Dukart J, Regen F, Kherif F, Colla M, Bajbouj M, Heuser I, et al. Electroconvulsive therapy-induced brain plasticity determines therapeutic outcome in mood disorders. Proc Natl Acad Sci. 2014;111:1156-61.

15. Oltedal L, Narr KL, Abbott C, Anand A, Argyelan M, Bartsch H, et al. Volume of the human hippocampus and clinical response following electroconvulsive therapy. Biol Psychiatry. 2018;84:574-81.

16. Argyelan M, Oltedal L, Deng Z-D, Wade B, Bikson M, Joanlanne A, et al. Electric field causes volumetric changes in the human brain. Elife. 2019;8:e49115.

17. van Oostrom I, van Eijndhoven P, Butterbrod E, van Beek MH, Janzing J, Donders $\mathrm{R}$, et al. Decreased cognitive functioning after electroconvulsive therapy is related to increased hippocampal volume: exploring the role of brain plasticity. J ECT. 2018;34:117-23.

18. Wang L, Hermens D, Hickie I, Lagopoulos J. A systematic review of resting-state functional-MRI studies in major depression. J Affect Disord. 2012;142:6-12.

19. Kaiser RH, Andrews-Hanna JR, Wager TD, Pizzagalli DA. Large-scale network dysfunction in major depressive disorder: a meta-analysis of resting-state functional connectivity. JAMA Psychiatry. 2015;72:603-11.

20. Beall EB, Malone DA, Dale RM, Muzina DJ, Koenig KA, Bhattacharrya PK, et al. Effects of electroconvulsive therapy on brain functional activation and connectivity in depression. J ECT. 2012;28:234-41.

21. Wang D, Li M, Wang M, Schoeppe F, Ren J, Chen $H$, et al. Individual-specific functional connectivity markers track dimensional and categorical features of psychotic illness. Mol Psychiatry. 2018:1-11.

22. Siddiqi SH, Taylor SF, Cooke D, Pascual-Leone A, George MS, Fox MD. Distinct symptom-specific treatment targets for circuit-based neuromodulation. Am J Psychiatry. 2020. appiajp201919090915.

23. Belge JB, Van Diermen L, Schrijvers D, Sabbe B, Constant E, de Timary P, et al. The basal ganglia: a central hub for the psychomotor effects of electroconvulsive therapy. J Affect Disord. 2020;265:239-46.

24. Leaver AM, Vasavada M, Kubicki A, Wade B, Loureiro J, Hellemann G, et al. Hippocampal subregions and networks linked with antidepressant response to electroconvulsive therapy. Mol Psychiatry. 2020:1-12.

25. Leaver AM, Vasavada M, Joshi SH, Wade B, Woods RP, Espinoza R, et al. Mechanisms of antidepressant response to electroconvulsive therapy studied with perfusion magnetic resonance imaging. Biol Psychiatry. 2019;85:466-76.

26. Mueller S, Wang D, Fox MD, Yeo BT, Sepulcre J, Sabuncu MR, et al. Individual variability in functional connectivity architecture of the human brain. Neuron. 2013;77:586-95.

27. Langs G, Golland P, Ghosh SS. Predicting activation across individuals with resting-state functional connectivity based multi-atlas label fusion. International
Conference on Medical Image Computing and Computer-Assisted Intervention: Springer; 2015

28. Wang D, Buckner RL, Fox MD, Holt DJ, Holmes AJ, Stoecklein S, et al. Parcellating cortical functional networks in individuals. Nat Neurosci. 2015;18:1853-60.

29. Laumann Timothy O, Gordon Evan M, Adeyemo B, Snyder Abraham Z, Joo Sung $J$, Chen $M-Y$, et al. Functional system and areal organization of a highly sampled individual human brain. Neuron. 2015;87:657-70.

30. Braga RM, Buckner RL. Parallel interdigitated distributed networks within the individual estimated by intrinsic functional connectivity. Neuron. 2017;95: 457-71.e5.

31. Li M, Wang D, Ren J, Langs G, Stoecklein S, Brennan BP, et al. Performing grouplevel functional image analyses based on homologous functional regions mapped in individuals. PLoS Biol. 2019;17:e2007032.

32. Brennan BP, Wang D, Li M, Perriello C, Ren J, Elias JA, et al. Use of an individuallevel approach to identify cortical connectivity biomarkers in obsessivecompulsive disorder. Biol Psychiatry. 2019;4:27-38.

33. Van Dijk KR, Sabuncu MR, Buckner RL. The influence of head motion on intrinsic functional connectivity MRI. Neuroimage. 2012;59:431-38.

34. Charlson F, Siskind D, Doi SA, McCallum E, Broome A, Lie DC. ECT efficacy and treatment course: a systematic review and meta-analysis of twice vs thrice weekly schedules. J Affect Disord. 2012;138:1-8.

35. Hamilton M. A rating scale for depression. J Neurol Neurosurg Psychiatry. 1960 23:56-62.

36. Rey A. L'examen clinique en psychologie [The clinical psychological examination] Paris: Presses Universitaires de France; 1964.

37. Thomas Yeo B, Krienen FM, Sepulcre J, Sabuncu MR, Lashkari D, Hollinshead M, et al. The organization of the human cerebral cortex estimated by intrinsic functional connectivity. J Neurophysiol. 2011;106:1125-65.

38. Van Dijk KR, Hedden T, Venkataraman A, Evans KC, Lazar SW, Buckner RL. Intrinsic functional connectivity as a tool for human connectomics: theory, properties, and optimization. J Neurophysiol. 2010;103:297-321.

39. Satterthwaite TD, Elliott MA, Gerraty RT, Ruparel K, Loughead J, Calkins ME, et al. An improved framework for confound regression and filtering for control of motion artifact in the preprocessing of resting-state functional connectivity data. Neuroimage. 2013;64:240-56.

40. Yan C-G, Cheung B, Kelly C, Colcombe S, Craddock RC, Di Martino A, et al. A comprehensive assessment of regional variation in the impact of head micromovements on functional connectomics. Neurolmage. 2013;76:183-201.

41. Zeng LL, Wang D, Fox MD, Sabuncu M, Hu D, Ge M, et al. Neurobiological basis of head motion in brain imaging. Proc Natl Acad Sci USA. 2014;111:6058-62.

42. Buckner RL, Krienen FM, Castellanos A, Diaz JC, Yeo BT. The organization of the human cerebellum estimated by intrinsic functional connectivity. J Neurophysiol. 2011;106:2322-45.

43. Ancelin ML, Isabelle C, Sylvaine A, Jerome M, Chantal M, Karen R, et al. Lifetime major depression and grey-matter volume. J Psychiatry Neurosci. 2018;44:45-53.

44. Jaworska N, Yang XR, Knott V, MacQueen G. A review of fMRI studies during visual emotive processing in major depressive disorder. World J Biol Psychiatry. 2015;16:448-71.

45. Finn ES, Shen X, Scheinost D, Rosenberg MD, Huang J, Chun MM, et al. Functional connectome fingerprinting: identifying individuals using patterns of brain connectivity. Nat Neurosci. 2015;18:1664.

46. Van Essen DC. A population-average, landmark-and surface-based (PALS) atlas of human cerebral cortex. Neuroimage. 2005;28:635-62.

47. Rottschy C, Langner R, Dogan I, Reetz K, Laird AR, Schulz JB, et al. Modelling neural correlates of working memory: a coordinate-based meta-analysis. Neuroimage. 2012;60:830-46

48. Sestieri C, Corbetta M, Romani GL, Shulman GL. Episodic memory retrieval, parietal cortex, and the default mode network: functional and topographic analyses. J Neurosci. 2011;31:4407-20.

49. Santangelo $V$, Bordier C. Large-scale brain networks underlying successful and unsuccessful encoding, maintenance, and retrieval of everyday scenes in visuospatial working memory. Front Psychol. 2019;10:233.

50. Drysdale AT, Grosenick L, Downar J, Dunlop K, Mansouri F, Meng Y, et al. Restingstate connectivity biomarkers define neurophysiological subtypes of depression. Nat Med. 2017;23:28-38.

51. Spreng RN, Sepulcre J, Turner GR, Stevens WD, Schacter DL. Intrinsic architecture underlying the relations among the default, dorsal attention, and frontoparietal control networks of the human brain. J Cogn Neurosci. 2013;25:74-86.

52. Moscovitch M, Cabeza R, Winocur G, Nadel L. Episodic memory and beyond the hippocampus and neocortex in transformation. Annu Rev Psychol. 2016;67:105-34.

53. Winocur G, Moscovitch M, Bontempi B. Memory formation and long-term retention in humans and animals: Convergence towards a transformation account of hippocampal-neocortical interactions. Neuropsychologia. 2010; 48:2339-56. 